\title{
Control of exuT Activity for Galacturonate Transport by the Negative Regulator ExuR in Erwinia chrysanthemi EC16
}

\author{
Venugopal Valmeekam, ${ }^{1}$ Yen-Lin Loh, ${ }^{2}$ and Michael J. D. San Francisco ${ }^{2}$ \\ ${ }^{1}$ Department of Biological Sciences, Texas Tech University, Lubbock 79409, U.S.A.; ${ }^{2}$ Affymetrix, 6550 \\ Vallejo Street, Emeryville, CA 94608, U.S.A. \\ Submitted 2 November 2000; Accepted 20 February 2001.
}

\begin{abstract}
The negative regulatory protein ExuR in Erwinia chrysanthemi regulates expression of the galacturonate uptake (exuT) and utilization (uxaA, uxaB, uxaC) genes. We cloned and determined the nucleotide sequence of the exuR gene from $E$. chrysanthemi EC16. Analysis of the deduced amino acid sequence indicates that this protein possesses a helix-turn-helix motif and belongs to the GntR family of transcriptional repressors. Northern blot analysis and studies with transcriptional fusions of exuT in wild-type and exuR mutant backgrounds indicate that exuT transcription is deregulated in the exuR strain in vivo and in planta. $\left[{ }^{14} \mathrm{C}\right]$-galacturonic acid uptake was constitutively high under inducing and noninducing conditions in the exuR mutant. Maximal exuT transcription activity was observed within $8 \mathrm{~h}$ of bacterial inoculation into potato tubers, well before any visible symptoms of disease were detected. This suggests that ExuT transport activity in E. chrysanthemi is important in the early stages of disease development.
\end{abstract}

Additional keywords: digalacturonate, gene regulation, pectin utilization, plant disease.

The plant pathogen Erwinia chrysanthemi causes soft rot disease by secreting large amounts of several different plant cell wall-degrading enzymes. Among these different walldegrading enzymes, pectate lyases have been identified as particularly important for E. chrysanthemi to macerate plant tissue (Collmer and Keen 1986; Keen and Tamaki 1986). Pectate lyases degrade pectate, a polymer of $\alpha$-1,4-linked galacturonic acid (GalUA), to generate GalUA dimers and multimers with varying degrees of polymerization (Preston et al. 1992). The products of extracellular pectate degradation are transported into the bacterial cell, where they undergo intracellular catabolism to generate inducer molecules for the expression of higher levels of pectate lyases and other cell wall-degrading enzymes (Hugouvieux-Cotte-Pattat et al. 1996). Therefore, uptake of pectate degradation products is required for the in-

Corresponding author: M. J. D. San Francisco;

Telephone: +1-806-742-2706; Fax: +1-806-742-2963;

E-mail: michael.sanfrancisco@ttu.edu duction of virulence factors and represents an important event in the initial stages of the plant-bacterial interaction, leading to the initiation and progression of disease.

Uptake of GalUA in E. chrysanthemi EC16 is induced by pectate and its degradation products, saturated digalacturonic acid (GalUA) ${ }_{2}$ and GalUA (San Francisco and Keenan 1993). The exuT gene encoding the transporter from this strain was cloned and sequenced, and the gene product was localized to the cytoplasmic membrane (Freeman and San Francisco, 1994; Haseloff et al. 1998). Mutants in exuT have a significantly lower capability to macerate potato tuber tissue than the wild-type parent (Haseloff et al. 1998). Information on the regulation of exuT expression has been restricted only to genetic analysis in E. chrysanthemi strains 3937 and B374 (Hugouvieux-Cotte-Pattat et al. 1983; Hugouvieux-CottePattat and Robert-Boudouy 1987). Wild-type E. chrysanthemi 3937 also can transport glucuronic acid (GulUA), an isomer of GalUA, with ExuT but cannot use GulUA as a sole carbon source for growth because this compound is unable to induce exuT expression (Hugouvieux-Cotte-Pattat et al. 1983). Spontaneous mutants capable of fermenting GulUA were isolated on the basis of their ability to grow on medium containing GulUA as a sole carbon source. This phenotype was attributed to the loss of a repressor function leading to the constitutive expression of the transport system, and the regulator was named ExuR, by analogy to the corresponding gene of Escherichia coli (Hugouvieux-Cotte-Pattat and Robert-Baudouy 1987; Nemoz et al. 1976; Portalier et al. 1980). Furthermore, $\mathrm{Mu}-$ lac insertions leading to constitutive uptake of $\left[{ }^{14} \mathrm{C}\right]-$ GulUA were isolated, and the mutations were assigned to the regulator of exuT expression. These studies represent indirect evidence for the role of exuR in the regulation of exuT. In view of the importance of GalUA transport and metabolism in the pathogenicity of E. chrysanthemi (Beaulieu and Van Gijsegem 1990; Haseloff et al. 1998), we wished to more clearly elucidate the mechanism of exuT regulation.

The gene encoding the presumptive ExuR was cloned (Freeman and San Francisco 1994), and the nucleotide sequence deposited under GenBank accession no. U35884. The deduced amino acid sequence of this gene indicates the presence of a polypeptide with a hydrophilic profile containing an N-terminal, helix-turn-helix motif (ProfileScan in the PROSITE database), which is characteristic of the GntR fam- 
ily of transcriptional regulators (Haydon and Guest 1991) (Fig. 1). This open reading frame is $777 \mathrm{bp}$ and predicted to encode a protein of 259 amino acids, with a calculated molecular mass of $31,081 \mathrm{kDa}$. A homology search of the DNA and protein databases with the deduced amino acid sequence revealed a strong homology with the E. coli ExuR (76\% identity), UxuR (48\% identity), and FarR (37\% identity) regulatory proteins, suggesting a regulatory function for the gene (Altschul et al. 1997). Examination of the $5^{\prime}$ upstream sequences of exuR indicate the presence of potential -10 and -35 consensus sequences (TATCAT and TTTCTG, respectively) corresponding to a prokaryotic promoter region. A putative ribosome binding sequence (GAGCAGG) (Shine and Dalgarno 1974) is located four bases upstream of the ATG start codon.

In order to study the role of exuR in the regulation of exuT expression, an E. chrysanthemi exuR strain was constructed by marker-exchange mutagenesis (Roeder and Collmer 1985). Previously, Northern blot analysis revealed that exuT transcripts are inducible in the presence of GalUA in wild-type $E$. chrysanthemi EC16 (Haseloff et al. 1998). In the E. chrysanthemi exuR strain, high levels of exuT transcripts were ob- served in uninduced (glycerol grown) and induced (GalUA grown) cultures (Fig 2). These levels of exuT transcripts were comparable to the levels in the induced wild-type cells and indicate the loss of regulatory control over exuT transcription. These results confirm that exuT expression is regulated by ExuR at the transcriptional level and that exuT transcription is constitutive in the exuR mutant.

Operon (transcription) fusions of exuT-lux were constructed in wild-type and exuR strains with the Tn7-lux system (Shen et al. 1992). Use of this system allows fusions to be inserted into the $\mathrm{Tn} 7$ att site on the chromosome, preventing insertion into functional genes. The strain used in these studies possesses a functional wild-type exuT allele. Southern blot analysis with the lux gene as a probe was carried out to confirm that only a single copy of the gene fusion was present on the chromosome. These single-copy operon fusions were used to obtain a detailed profile of exuT expression in relation to different phases of bacterial growth in culture and during plant tissue maceration. In vivo studies were carried out by monitoring bioluminescence of cells grown in glycerol or GalUA at regular time points for a period of $15 \mathrm{~h}$ (Fig. 3). Starter cultures were grown in M9 glycerol overnight before inoculation

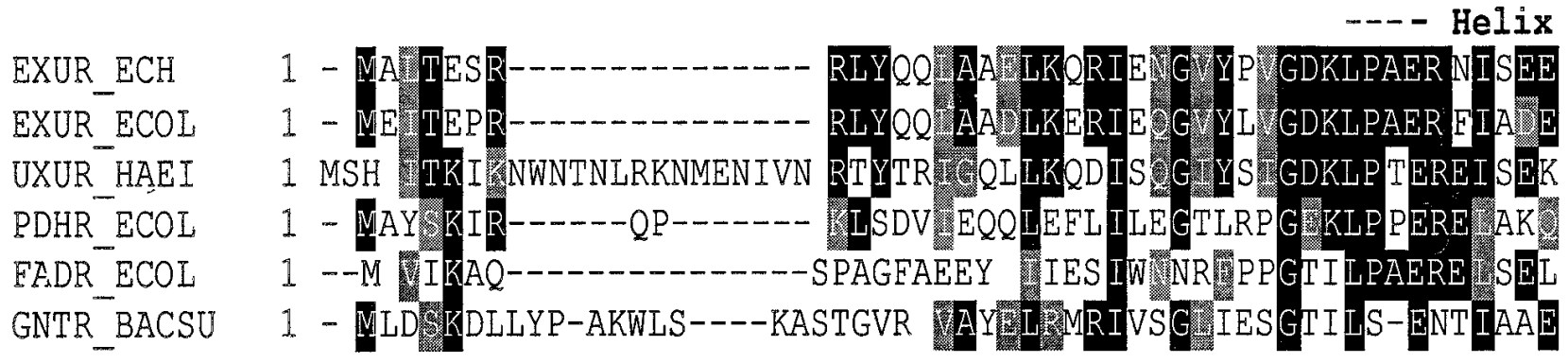

-Turn-Helix motif--
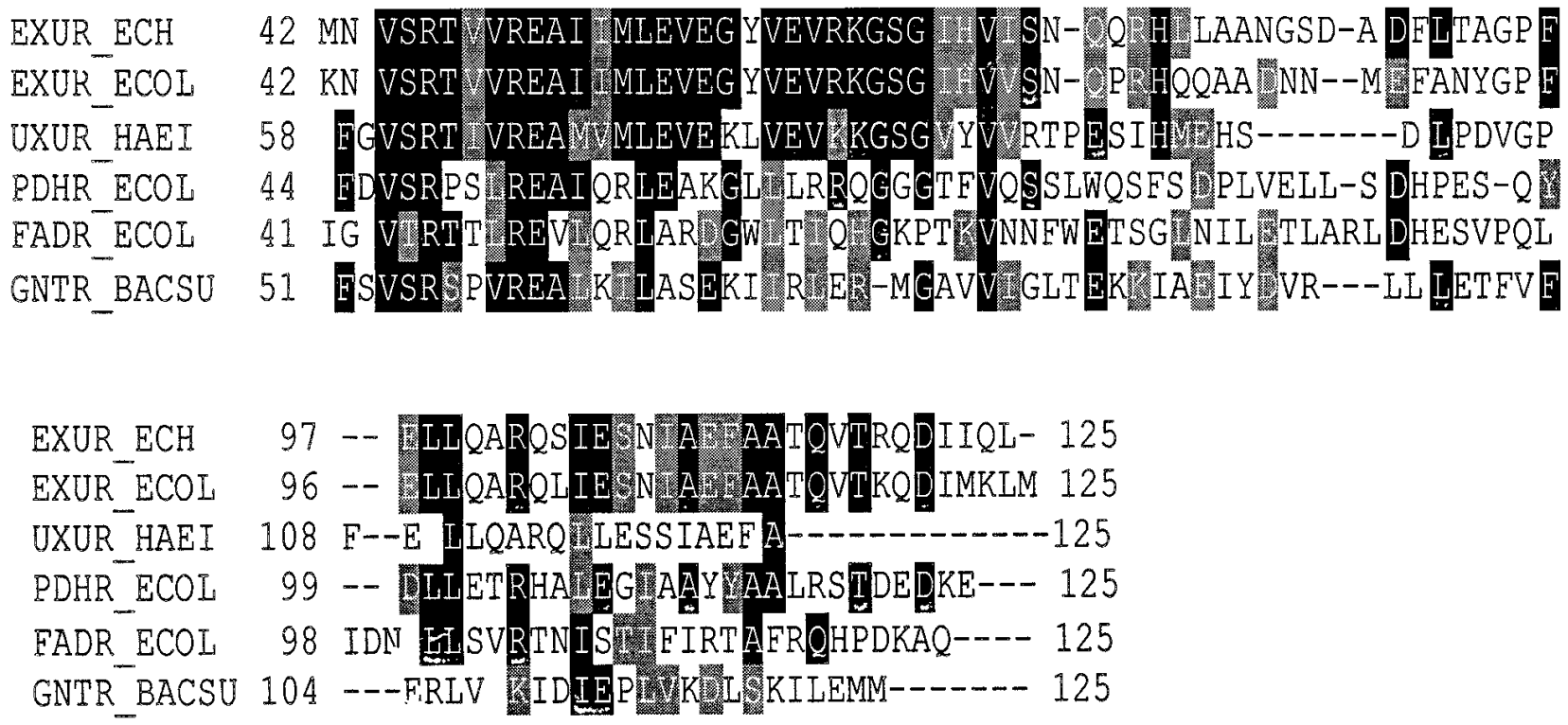

Fig. 1. Alignment of the N-terminal region of ExuR with homologs of the GntR family of regulatory proteins. EXUR_ECH: Repressor for galacturonate uptake and utilization genes from Erwinia chrysanthemi. EXUR_ECOL: Repressor for galacturonate uptake and utilization genes from Escherichia coli. UXUR_HAE1: Repressor glucuronate transport and utilization genes from Haemophilus influenzae. PDHR_ECOL: Repressor for pyruvate dehydrogenase from E. coli. FADR_ECOL: Fatty acid-responsive gene from E. coli. GNTR_BACSU: Repressor for gluconate transport and utilization genes from Bacillus subtilis. 
into media to monitor exuT expression. Wild-type cells grown on glycerol (uninduced) had basal levels of exuT-lux activity, reaching a maximal value of $5.3 \times 10^{7} \mathrm{cpm}$ after $10 \mathrm{~h}$ (luciferase activity on a cell-density basis) (Fig. 3). When these cells were grown in GalUA as an inducer, luciferase activity increased rapidly and attained levels approximately 2.5 times the basal values observed with uninduced cultures (solid circles). Highest levels of expression were observed during the mid-log phase and declined rapidly to basal levels before the onset of stationary phase. These results indicate that exuT-lux expression is induced by GalUA in wild-type cells. The expression profile of the exuT-lux fusion was different in the exuR strain compared with the wild type. Luciferase activity of the uninduced exuR mutant (Fig. 3) reached levels comparable to the induced wild-type cultures. The peak value was slightly higher than the wild-type cells grown in GalUA, and exuT-lux expression remained relatively high, even in stationary phase cultures. This suggests that exuT-lux expression is deregulated in the exuR mutant. The expression profile of exuT transcription in the exuR mutant in the presence of GalUA (Fig. 3) was unexpected, comprising a distinct "lag phase" before reaching higher levels in early stationary phase cultures. The reason for this effect is unclear and may represent additional controls such as $\mathrm{KdgR}$ on this important transport system. Sequence analysis of the $5^{\prime}$ exuT region indicated the presence of a conserved $\mathrm{KdgR}$ binding box (Haseloff et al. 1998). How this protein or any others interact in exuT expression can only be speculated at this time.

The relevance of exuR during potato tuber infections was studied by monitoring exuT-lux expression in wild-type and exuR strains. Cored potato cubes were inoculated with approximately $10^{7}$ bacterial cells and incubated in scintillation vials in a humid environment at $30^{\circ} \mathrm{C}$. Luciferase activity was

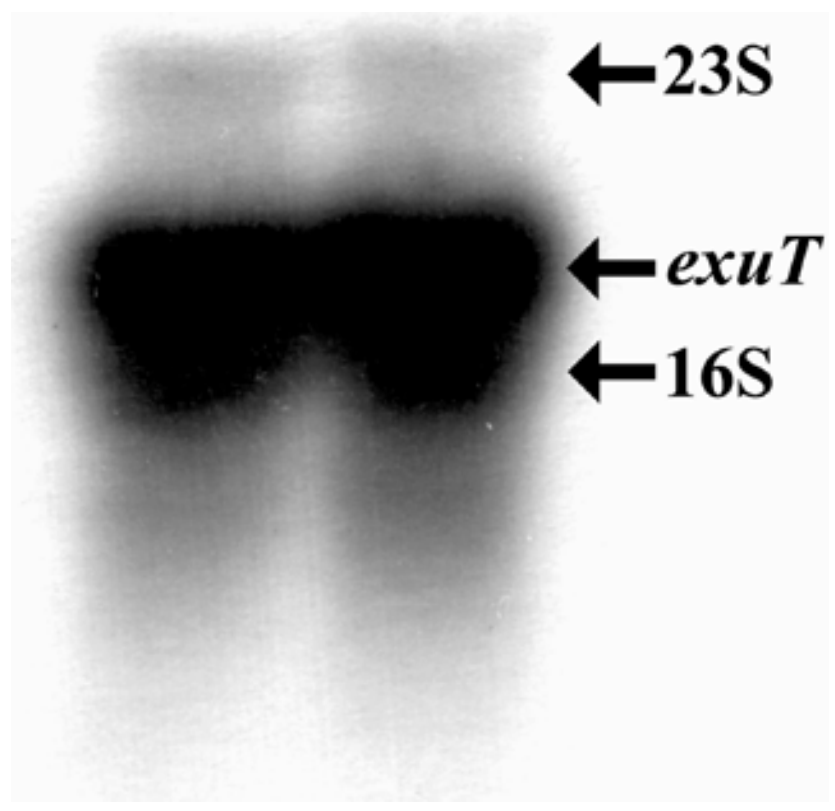

Fig 2. Analysis of exuT transcription by Northern blot hybridization. Total RNA isolated from Erwinia chrysanthemi exuR mutant grown on M9 salts medium with $0.2 \%$ glycerol (uninduced, lane 1) or galacturonic acid (induced, lane 2) was separated on a $1.2 \%$ formaldehyde gel. A 1.1-kb KpnI fragment of the exuT gene was used as the probe. monitored at regular intervals in a scintillation counter. The strain bearing the exuR mutation resulted in an earlier onset of maceration symptoms compared to the wild type, and luciferase activity in the exuR strain increased rapidly and reached higher levels (approximately 1.5 fold) than the wildtype strain (data not shown). Thus, the exuR mutation appears to influence the rate and extent of potato tissue maceration. Observations also were made to assess the temporal expression pattern of exuT during plant tissue maceration with potato tuber tissue. Transcription of exuT increased rapidly over the first $5 \mathrm{~h}$, peaked at $7 \mathrm{~h}$, and declined to basal levels at $34 \mathrm{~h}$ postinoculation (Fig. 4). Interestingly, initial visible symptoms of potato tuber maceration were observed at approximately 10 to $12 \mathrm{~h}$ postinoculation, and host tissue was macerated thoroughly at 22 to $34 \mathrm{~h}$ postinoculation. Previous studies with a pelE-lux fusion in E. chrysanthemi inoculated into potato tubers showed that pelE transcription increased continually and peaked at approximately $20 \mathrm{~h}$ postinoculation (Gold et al. 1992; Shen et al. 1992). Our data strongly suggest that exuT transcription is important in the very early stages of the plantmicrobe interaction. One possible explanation for the lower levels of exuT transcription following visible signs of tissue maceration could be the availability of alternate carbon sources, some of which may catabolite repress transcription of the gene.

To observe the effect of the exuR mutation on ExuT transport activity, $\left[{ }^{14} \mathrm{C}\right]$-GalUA uptake was studied in the wild-type and exuR mutant. $\left[{ }^{14} \mathrm{C}\right]$-GalUA uptake activity remained at basal levels in wild-type cells grown under noninducing conditions (Fig. 5) but increased approximately twofold in cells grown in the presence of the inducer. These results are consistent with previous reports indicating inducible GalUA uptake

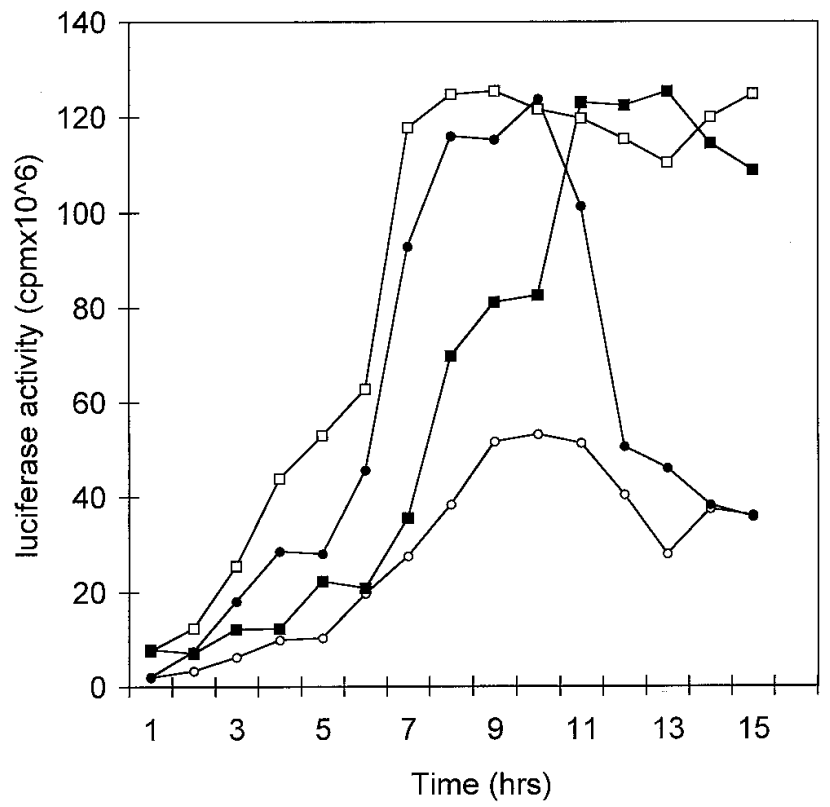

Fig. 3. In vivo luciferase activity of the wild-type and $e x u R$ strains containing the exuT-lux operon fusions. Circles denote the wild-type strain and squares represent the exuR mutant. Open and solid symbols represent data obtained from cultures grown in M9 salts with $0.2 \%$ glycerol (uninduced) and $0.2 \%$ galacturonic acid (induced), respectively. Values represent the average of three replicate treatments. The experiment was repeated three times. 


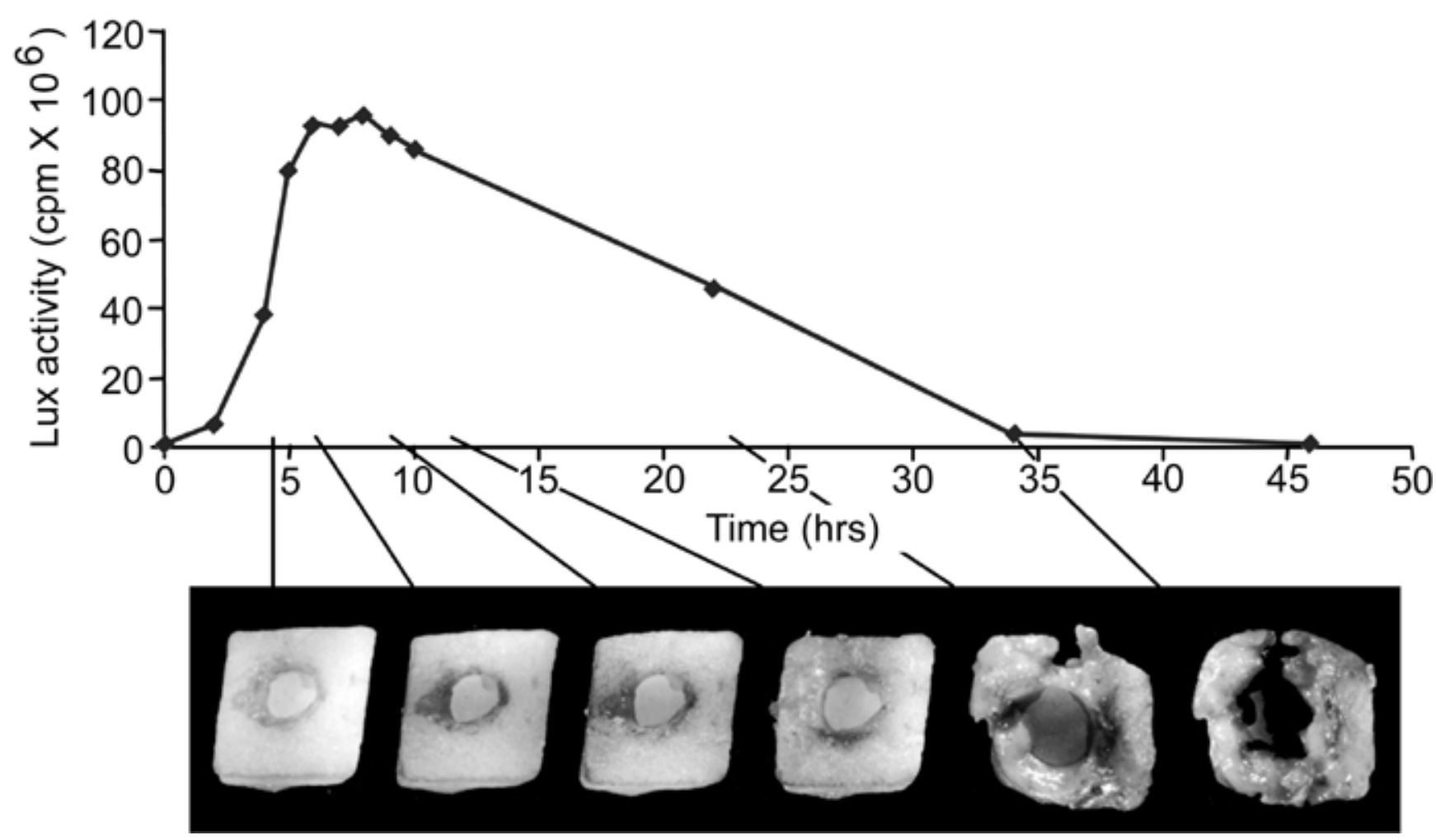

Fig 4. Temporal expression of exuT during potato tuber maceration. Bacteria carrying the exuT-luxAB fusion were inoculated into cored potato cubes and incubated in a humid environment at $30^{\circ} \mathrm{C}$. These data represent an average of 19 replications over the time course. Pictures represent a visualization of the progression of tissue maceration over the time course of the experiment.

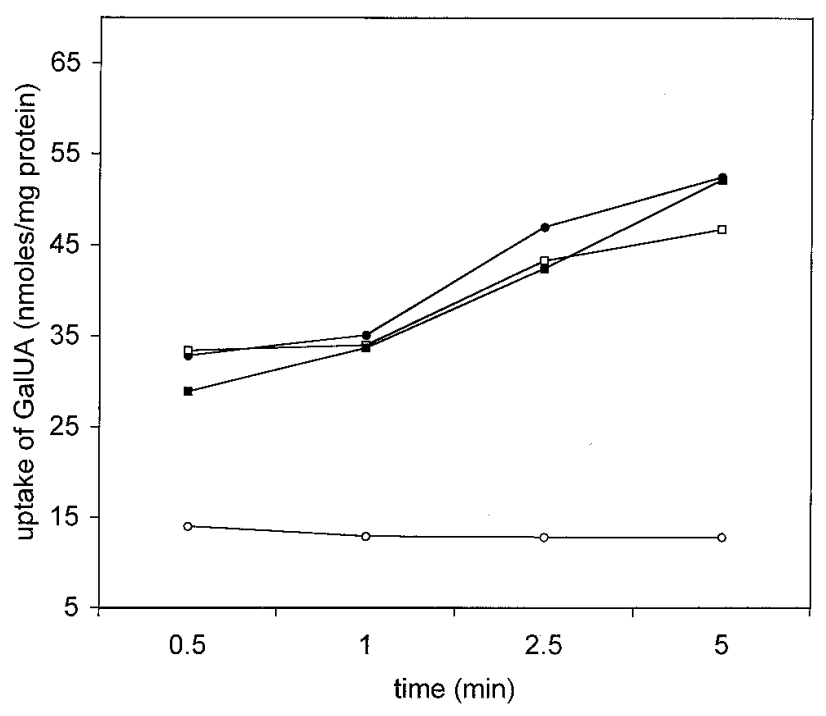

Fig. 5. Uptake of $\left[{ }^{14} \mathrm{C}\right]$ - galacturonic acid (GalUA) of the wild-type and exuR mutant. Circles and squares denote the wild-type and exuR mutant, respectively. The cultures were grown in $\mathrm{M} 9$ salts with $0.2 \%$ glycerol (uninduced) or $0.2 \%$ GalUA (induced) and are denoted in the graph by open and closed symbols, respectively. The data points are average $(+/-$ standard error) of three independent experiments.

activity in wild-type E. chrysanthemi EC16. In the exuR mutant, $\left[{ }^{14} \mathrm{C}\right]$-GalUA uptake activity was constitutively high under inducing and noninducing conditions. These results suggest that GalUA uptake is deregulated in the exuR mutant, which indicates a loss-of-repressor function. Results presented in this paper provide convincing evidence that exuT expression correlates with the early events of plant-microbe interactions and that ExuR is the major regulator of exuT transcription. Studies are under way to identify exuT DNA sequences that interact with the repressor, ExuR. Identification of these sequences through DNA mobility shift and footprint analyses will aid in the identification of other genes that may be regulated by ExuR and other members of the GntR family of repressors.

\section{ACKNOWLEDGMENTS}

This work was supported by NRI grant 94-3703-0547 from the U.S. Department of Agriculture to M. J. D. San Francisco and a seed grant from Texas Tech University. Y.-L. Loh was supported by the National Science Foundation Research Experiences for Undergraduates Program (DBI-9322342) and a Howard Hughes Medical Institute grant through the Undergraduate Biological Sciences Education Program to Texas Tech University. R. Deshippe is thanked for assistance with figures.

\section{LITERATURE CITED}

Altschul, S. F., Stephen, F., Thomas, L. M., Alejandro, A. S., Zang, J., Zhang, Z., Miller, W., and Lipman, D. J. 1997. Gapped BLAST and PSI-BLAST: A new generation of protein database search programs. Nucleic Acids Res. 25:3389-3402.

Beaulieu, C., and Van Gijsegem, F. 1990. Identification of plantinducible genes in Erwinia chrysanthemi 3937. J. Bacteriol. 172:1569-1575.

Collmer, A., and Keen, N. T. 1986. The role of pectic enzymes in plant pathogenesis. Annu. Rev. Phytopathol. 24:383-409.

Freeman, T. L., and San Francisco, M. J. D. 1994. Cloning of a galactu- 
ronic acid uptake gene from Erwinia chrysanthemi EC16. FEMS Microbiol. Lett. 118:101-106.

Gold, S., Nishio, S., Tsuyumu, S., and Keen, N. T. 1992. Analysis of the pelE promoter in Erwinia chrysanthemi EC16. Mol. Plant-Microbe Interact. 5:170-178.

Haseloff, B. J., Freeman, T. L., Valmeekam, V., Melkus, M. W., Oner, F., Valachovic, M. S., and San Francisco, M. J. D. 1998. The exuT gene of Erwinia chrysanthemi EC16: Nucleotide sequence, expression, localization, and relevance of the gene product. Mol. Plant-Microbe Interact. 11:270-276.

Haydon, D. J., and Guest, J. R. 1991. A new family of bacterial regulatory proteins. FEMS Microbiol. Lett. 79:291-296.

Hugouvieux-Cotte-Pattat, N., and Robert-Baudouy, J. 1987. Hexuronate catabolism in Erwinia chrysanthemi. J. Bacteriol. 169:12231231.

Hugouvieux-Cotte-Pattat, N., Quesneau, Y., and Robert-Baudouy, J. 1983. Aldohexuronate transport system in Erwinia carotovora. J. Bacteriol. 154:663-668.

Hugouvieux-Cotte-Pattat, N., Condemine, G., Nasser, W., and Reverchon, S. 1996. Regulation of pectinolysis in Erwinia chrysanthemi. Annu. Rev. Microbiol. 50:213-257.

Keen, N. T., and Tamaki, S. 1986. Structure of two pectate lyase genes from Erwinia chrysanthemi EC16 and their high level expression in Escherichia coli. J. Bacteriol. 168:595-606.

Nemoz, G., Robert-Baudouy, J., and Stoeber, F. 1976. Physiological and genetic regulation of the aldohexuronate transport system. J. Bacteriol. 127:706-718.

Portalier, R., Robert-Boudouy, J., and Stoeber, F. 1980. Regulation of Escherichia coli K-12 hexuronate system genes: exu regulon. J. Bacteriol. 143:1095-1107.

Preston, J. F., III, Rice, J. D., Ingram, L. O., and Keen, N. T. 1992. Differential depolymerization mechanisms of pectate lyases secreted by Erwinia chrysanthemi EC16. J. Bacteriol. 174:2039-2942.

Roeder, D. L., and Collmer, A. 1985. Marker-exchange mutagenesis of a pectate lyase isozyme gene in Erwinia chrysanthemi. J. Bacteriol. 164:51-56

San Francisco, M. J. D., and Keenan, R. W. 1993. Uptake of galacturonic acid in Erwinia chrysanthemi EC16. J. Bacteriol. 175:4263-4265.

Shen, H., Gold, S. E., Tamaki, S. J., and Keen, N. T. 1992. Construction of a Tn7-lux system for gene expression studies in Gram-negative bacteria. Gene 122:27-34.

Shine, J., and Dalgarno, L. 1974. 3'-terminal sequence of Escherichia coli 16S ribosomal RNA: Complementarity to nonsense triplets and ribosome binding sites. Proc. Natl. Acad. Sci. USA 71:1342-1346. 